\title{
镰翅羊耳蒜中的新黄酮苷类化合物
}

$$
\text { 吴润黄帅*单连海李遂焰魏屹周先礼* }
$$

(西南交通大学生命科学与工程学院 成都 610031)

\begin{abstract}
摘要 利用多种分离方法对镰翅羊耳蒜的化学成分进行研究, 从其全草的乙醇提取物中分离得到两个化合物, 经过 HR-ESIMS, $1 \mathrm{D}$ 和 $2 \mathrm{D}$ NMR 等波谱技术鉴定了结构，分别为山奈酚 3- $O-\alpha-L$ - 鼠李糖-7- $O$-[6-(4-差基-3-异戊二烯基苯甲酸

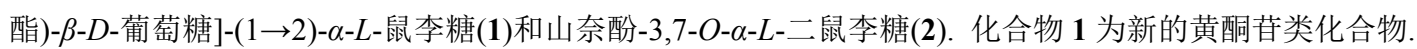

关键词 羊耳蒜属; 镰翅羊耳蒜; 黄酮苷
\end{abstract}

\section{A New Flavone Glycoside from Liparis bootanensis}

\author{
Wu, Run Huang, Shuai* Shan, Lianhai $\quad$ Li, Suiyan $\quad$ Wei, Yi $\quad$ Zhou, Xianli* \\ (School of Life Science and Engineering, Southwest Jiaotong University, Chengdu 610031)
}

\begin{abstract}
A new flavonoid glycoside, kaempferol-3-O- $\alpha-L$-rhamnopyranoside-7-O-[6-(4-hydroxy-3-prenybenzoic ester)- $\beta$ -

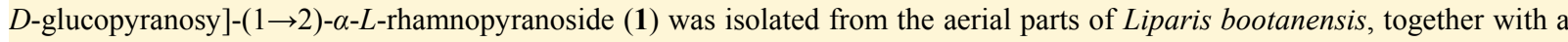
known compound kaempferol-3,7-di-O- $\alpha$ - L-rhamnopyranoside (2) by silica gel column and Pre-HPLC. Their structures were elucidated on the basis of extensive spectroscopic analysis, including HR-ESIMS, 1D and 2D NMR experiments.
\end{abstract}

Keywords Liparis L. C. Rich; Liparis bootanensis; flavone glycoside

羊耳蒜属(Liparis L. C. Rich)为兰科植物, 全球约 250 种, 我国有 45 种, 约 19 种产自台湾, 其余主要分布 在西南地区 ${ }^{[1]}$. 其中多种植物如羊耳蒜、见血青、小花 羊耳蒜、大花羊耳蒜等在历代本草中都有记载, 民间多 以全草入药 ${ }^{[2]}$. 该属植物主要含生物碱、酚酸、黄酮及 苷、萜、甾醇等结构类型的成分 ${ }^{[2]}$. 镰翅羊耳蒜 (Liparis bootanensis Griff.)广泛分布于江西南部、福建、台湾、 广东、海南、广西、四川西南部等地, 附生于海拔 800-2300 米的林中或山谷岩石上，具有解热止咳、止血、 止痛作用, 主治肺热咳嗽、痰中带血、月经过多、风湿 腰腿痛等症 ${ }^{[3 \sim 4]}$. 目前还未见镰翅羊耳蒜化学成分及生 物活性的报道. 为更好地开发利用羊耳蒜属植物的药用 资源, 我们课题组首次系统开展镰翅羊耳蒜化学成分研 究，通过正、反相硅胶柱层析、制备高效液相色谱等技 术从其乙醇提取物的正丁醇部分中分离纯化得到两个 单体化合物, 运用 HR-ESIMS, 1D 和 2D NMR 等多种光 谱手段确定了它们的结构(图 1), 分别为山奈酚 3-O- $\alpha-L-$
鼠李糖-7- $O$-[6-(4-着基-3-异戊二烯基苯甲酸酯)- $\beta$ - $D$-葡

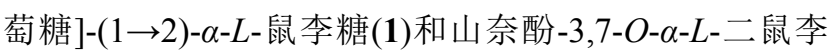
糖(2). 化合物 1 是首次报道的含有 4-羟基-3-异戊二烯基 苯甲酸酯片段的黄酮苷类化合物.

\section{1 结果与讨论}

化合物 1 为黄色粉末, m. p. $213{ }^{\circ} \mathrm{C}$, 三氯化铁反应 呈阳性; $[\alpha]_{\mathrm{D}}^{20}-28.8$ (c $\left.0.50, \mathrm{CH}_{3} \mathrm{OH}\right)$; HR-ESIMS 显示 化合物分子式为 $\mathrm{C}_{45} \mathrm{H}_{52} \mathrm{O}_{21}(\mathrm{~m} / \mathrm{z}$ : 929.3078; calcd for $[\mathrm{M}+\mathrm{H}]^{+}$929.3079), 不饱和度为 20 . 红外光谱显示有 羟基 $\left(3408 \mathrm{~cm}^{-1}\right)$ 、羰基(1704, $\left.1655 \mathrm{~cm}^{-1}\right)$ 和芳环 $(1512$, $\left.1602 \mathrm{~cm}^{-1}\right)$ 的存在. ${ }^{1} \mathrm{H}$ NMR 谱中氢信号 $\left[\delta_{\mathrm{H}} 6.20(\mathrm{~d}, J=\right.$ $1.8 \mathrm{~Hz}, 1 \mathrm{H}) ; 6.42$ (d, $J=1.8 \mathrm{~Hz}, 1 \mathrm{H}) ; 7.81$ (d, $J=9.0 \mathrm{~Hz}$, $2 \mathrm{H}) ; 6.95(\mathrm{~d}, J=9.0 \mathrm{~Hz}, 2 \mathrm{H})],{ }^{13} \mathrm{C} \mathrm{NMR}$ 谱中的羰基信号 $\left[\delta_{\mathrm{C}} 179.9(\mathrm{~s})\right]$, 推测该化合物是黄酮醇类 ${ }^{[5]} .{ }^{13} \mathrm{C}$ NMR 数 据中的 15 个碳信号 $\left[\delta_{\mathrm{C}} 159.8(\mathrm{~s}), 136.6(\mathrm{~s}), 179.9(\mathrm{~s})\right.$,

*E-mail: xxbiochem@163.com; shuaih@home.swjtu.edu.cn

Received April 26, 2016; revised June 24, 2016; published online July 13, 2016.

Project supported by the National Natural Science Foundation of China (No. 81274193), the Small Molecular Natural Medicine Innovation Team of Sichuan Province (No. 15TD0048), the Fundamental Research Funds for Central Universities (No. 2682015YXZT03), the Project of Science and Technology Bureau of Chengdu City (No. 2015-HM01-00041-SF) and the Southwest Jiaotong University Graduate Students Creative Experiment (No. YC201510104).

国家自然科学基金(No. 81274193)、四川省小分子天然药物创新团队(No. 15TD0048)、中央高校基础科研业务费专项(No. 2682015YXZT03)、成都市 科技项目(No. 2015-HM01-00041-SF)和西南交通大学 2015 年研究生创新实验实践(No. YC201510104)资助项目. 


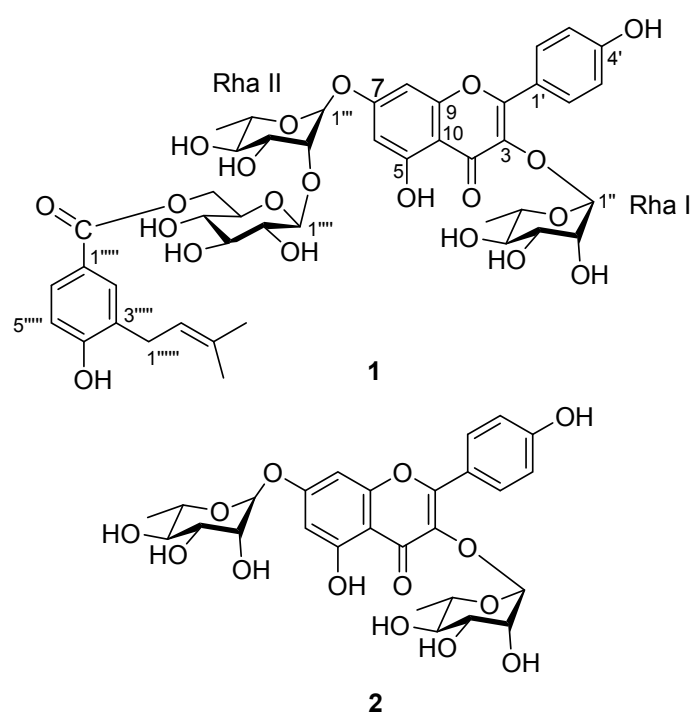

图 1 化合物 1 和 2 结构图

Figure 1 Structures of compounds 1 and 2

162.9 (s), 100.5 (d), 163.0 (s), 95.4 (d), 158.0 (s), 107.7 (s), $122.5(\mathrm{~s}), 132.0 \times 2$ (d), $116.6 \times 2$ (d), 161.7 (s)], 与山奈 酚碳谱数据基本一致, 区别在于 C-3 和 C-7 的化学位移 分别向高场移动了 $\delta 3$ 和 5 , 推断该化合物中的黄酮苷 元为山奈酚, 且 3,7 位形成了糖苷 ${ }^{[6]}$. 化合物 $\mathbf{1}$ 通过酸水 解得到糖部分, 衍生化后, 再通过 $\mathrm{GC}$ 与标准物比对, 结合 ${ }^{1} \mathrm{H}$ NMR 谱 $\left[\delta_{\mathrm{H}} 5.41(\mathrm{~d}, J=1.8 \mathrm{~Hz}, 1 \mathrm{H}), 5.17(\mathrm{~d}, J=\right.$ $1.8 \mathrm{~Hz}, 1 \mathrm{H}), 4.54(\mathrm{~d}, J=7.8 \mathrm{~Hz}, 1 \mathrm{H})$ ], 推测该化合物存在

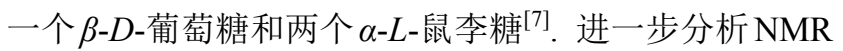
图谱可知, 化合物中存在一个异戊二烯基段片 $\left[\delta_{\mathrm{C}} 17.9\right.$ (q), 25.9 (q), $123.2(\mathrm{~s}), 29.1(\mathrm{t}), 133.6(\mathrm{~s})$ ] 和一个 3,4 二取 代的苯甲酸结构片段 $\left[\delta_{\mathrm{H}} 6.51(\mathrm{~d}, J=8.4 \mathrm{~Hz}, 1 \mathrm{H}), 7.68\right.$ (dd, $J=8.4,1.8 \mathrm{~Hz}, 1 \mathrm{H}$ ), 7.69 (br s, $1 \mathrm{H}$ ); $\delta_{\mathrm{C}} 115.2$ (d), 121.6 (s), 129.0 (s), 130.2 (d), 132.3 (d), 161.4 (s), 168.3 (s)], 结合 2D NMR 和文献[8]对比, 确定化合物中存在 一个 4-羟基-3-异戊二烯基苯甲酸酯结构片段. $\mathrm{HMBC}$ 图 谱(图 2)中鼠李糖 Rha I 的端基氢 H-1" $\left[\delta_{\mathrm{H}} 5.41\right.$ (d, $J=1.8$ $\mathrm{Hz}, 1 \mathrm{H})]$ 与苷元山奈酚的 C-3 $\left[\delta_{\mathrm{C}} 136.6(\mathrm{~s})\right]$ 相关, 鼠李糖 Rha II 的端基氢 H-1"' $\left[\delta_{\mathrm{H}} 5.17(\mathrm{~d}, J=1.8 \mathrm{~Hz}, 1 \mathrm{H})\right]$ 与苷元 的 C-7 [ $\delta_{\mathrm{C}} 163.0(\mathrm{~s})$ ] 相关, 证明鼠李糖 Rha I 和鼠李糖 Rha II 分别与黄酮醇母核的 C-3, C-7 位相连; 葡萄糖 Glc I 端基氢 H-1"' $\left[\delta_{\mathrm{H}} 4.54(\mathrm{~d}, J=7.8 \mathrm{~Hz}, 1 \mathrm{H})\right]$ 与鼠李糖 Rha II 中 C-2"' $\left[\delta_{\mathrm{C}} 83.3(\mathrm{~d})\right]$ 相关, 可知葡萄糖位于鼠李糖 Rha II 的 C-2"'位; 此外，葡萄糖 Glc I 中的 H-6"'" $\left[\delta_{\mathrm{H}} 4.51(\mathrm{dd}\right.$, $J=8.4,12.0 \mathrm{~Hz}, 1 \mathrm{H}), 4.61(\mathrm{dd}, J=2.4,12.0 \mathrm{~Hz}, 1 \mathrm{H})]$ 与 4羟基-3-异戊二烯基苯甲酯结构中羰基 $\left[\delta_{\mathrm{C}} 168.3(\mathrm{~s})\right]$ 相关. 综上所述, 化合物 1 的结构鉴定为山奈酚 3- $O-\alpha-L$ - 鼠李 糖-7- $O$ - $[6$-(4-羟基-3-异戊二烯基苯甲酸酯) $-\beta$ - $D$ - 葡萄

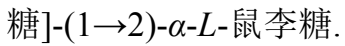

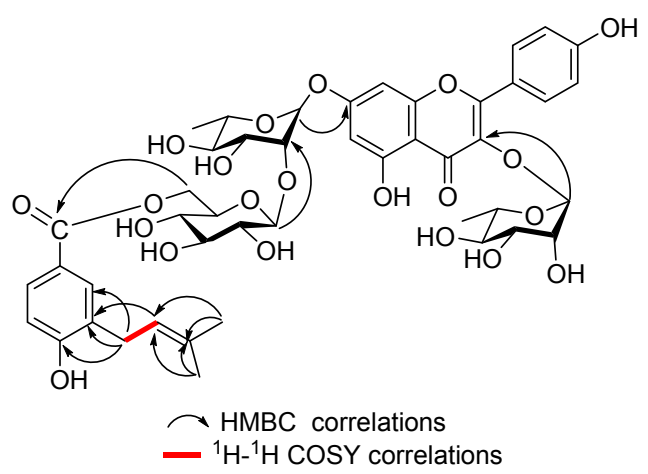

图 2 化合物 1 的关键 $\mathrm{HMBC}$ 与 ${ }^{1} \mathrm{H}-{ }^{1} \mathrm{H}$ COSY 相关 Figure 2 Key $\mathrm{HMBC}$ and ${ }^{1} \mathrm{H}-{ }^{1} \mathrm{H}$ COSY correlations of compound 1

化合物 $\mathbf{2}$ 核磁数据与文献[9]一致, 故将它鉴定为山 奈酚-3,7-O- $\alpha-L-$ 二鼠李糖.

\section{2 实验部分}

\section{1 仪器与试剂}

核磁共振谱仪 Brucker AVANCE DRX-600; 超高效 液相色谱仪(ACQUITY UPLC I-Class)与四级杆飞行时 间质谱(Xevo G2-S QTof) (waters 公司); NP700C 制备液 相色谱仪(江苏汉邦科技有限公司), 制备柱为 Herera ODS-2 $(10 \mu \mathrm{m}, 50 \mathrm{~mm} \times 250 \mu \mathrm{mm})$; 旋光光度仪 (Perkin-Elmer Polarimeter 341); 红外光谱仪 (Thermo Fisher Nicolet 6700); 气相色谱仪(Agilent 6890N); 差示 扫描量热仪(耐驰 DSC 214 Polyma); 层析用硅胶 $\mathrm{G}$ 和 $\mathrm{H}$ (青岛海洋化工厂). 其余试剂为分析纯.

\section{2 实验样品}

镰翅羊耳蒜于 2014 年 8 月采自广西百合, 经西南交 通大学宋良科副教授鉴定为镰翅羊耳蒜 (L. bootanensis), 标本(No. 361520140802Y)保存于西南交通大学生命科 学与工程学院.

\section{3 提取与分离}

镰翅羊耳蒜 $8 \mathrm{~kg}$ 用 3 倍体积的 $95 \%$ 乙醇冷浸提取 3 次, 提取液减压浓缩得 $1.15 \mathrm{~kg}$ 褐色粘稠状浸膏. 取 1.1 $\mathrm{kg}$ 浸膏悬浮于 $50{ }^{\circ} \mathrm{C}$ 的热水中, 依次用石油醚萃取 (1 $\mathrm{L} \times 3)$ 、乙酸乙酯萃取 $(1 \mathrm{~L} \times 3)$ 、正丁醇萃取 $(1 \mathrm{~L} \times 3)$, 减 压浓缩后得到石油醚浸膏 A (200 g)、乙酸乙酯浸膏 B $(260 \mathrm{~g})$ 、正丁醇浸膏 C (360 g). 正丁醇浸膏 C 经 D-101 大孔树脂色谱，分别用水、30\%乙醇、 $60 \%$ 乙醇、 $95 \%$ 乙醇洗脱得 $\mathrm{C}_{1} \sim \mathrm{C}_{3}$ 三个部分, $\mathrm{C}_{1}(90 \mathrm{~g})$ 部分经过正相硅 胶柱色谱(乙酸乙酯-甲醇, $V: V=50: 1 \rightarrow 0: 1$ ) 分为 $\mathrm{C}_{1-1} \sim \mathrm{C}_{1-6}$ 六个部分. $\mathrm{C}_{1-1}(3 \mathrm{~g})$ 部分经过正相硅胶柱色谱 
(氯仿一甲醇, $V: V=15: 1 \rightarrow 1: 1$ ) 得到 $\mathrm{C}_{1-1-1} \sim \mathrm{C}_{1-1-5}$ 部 分. $\mathrm{C}_{1-1-5}$ 部分经过正相硅胶柱色谱(乙酸乙酯-甲醇, $V$ : $V=50: 0.5 \rightarrow 1: 1$ )得到 $\mathrm{C}_{1-1-5-1}$ 和 $\mathrm{C}_{1-1-5-2}$ 部分. $\mathrm{C}_{1-1-5-2}$ 经 过正相硅胶柱色谱(乙酸乙酯-甲醇-水, $V: V: V=30$ : $0.5: 0.5$ ) 和高效液相色谱制备 (甲醇-水, $V: V=1:$ $4 \rightarrow 1: 1$, 流速 $10 \mathrm{~mL} / \mathrm{min}$, 检测波长 $240 \mathrm{~nm}$ ) 得到化合 物 $\mathbf{1}(12 \mathrm{mg})$ 和化合物 $\mathbf{2}(200 \mathrm{mg})$.

\section{4 酸水解}

将化合物 $1(2 \mathrm{mg})$ 溶于 $1.5 \mathrm{~mL}$ 的 $4 \mathrm{~mol} / \mathrm{L}$ 盐酸的二 氧六环溶液中, 加热到 $90{ }^{\circ} \mathrm{C}$ 保持 $4 \mathrm{~h}$. 冷却后, 用水 $(3$ $\mathrm{mL}$ )稀释, 再用 $2 \mathrm{~mol} / \mathrm{L}$ 氨水中和, 最后用氯仿 $(3 \mathrm{~mL} \times$ 3 )萃取, 剩余水部分用氮气吹干, 加入三甲基硅烷 (0.1 $\mathrm{mL})$ 和吡啶 $(0.2 \mathrm{~mL})$ 在 $60{ }^{\circ} \mathrm{C}$ 下搅拌 $20 \mathrm{~min}$. 将得到的溶 液用色谱柱为 L-CP-Chirasil-Val $(0.32 \mathrm{~mm} \times 25 \mathrm{~m})$ 的 GC 进行分析, 设置注样器温度和检测器温度为 $200{ }^{\circ} \mathrm{C}$, 柱 箱温度为 $60{ }^{\circ} \mathrm{C}$ 维持 $2 \mathrm{~min}$, 然后以 $15{ }^{\circ} \mathrm{C} / \mathrm{min}$ 持续升温 到 $250{ }^{\circ} \mathrm{C}$ 检测样品. 将样品与通过相同条件处理的 $\beta$ - $D$-葡萄糖(13.04 $\mathrm{min}$ )和 $\alpha$ - $L$-鼠李糖(11.10 min)的标准 品具有相同的保留时间, 且峰面积比接近 $1: 2$. 由此, 推断该化合物中含有一个 $\beta$ - $D$-葡萄糖和两个 $\alpha-L$ - 鼠李 糖 ${ }^{[7]}$.

\section{5 结构测定}

山奈酚-3- $O-\alpha-L$ - 鼠李糖-7-O-[6-(4-羟基-3-异戊二烯

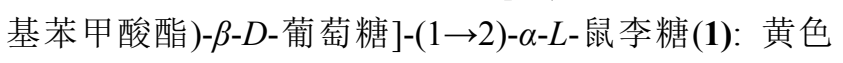
粉末. m.p. $213{ }^{\circ} \mathrm{C} ;[\alpha]_{\mathrm{D}}^{20}-28.8\left(\right.$ c $\left.0.50, \mathrm{CH}_{3} \mathrm{OH}\right) ;{ }^{1} \mathrm{H}$ NMR (600 MHz, CD $\left.{ }_{3} \mathrm{OD}\right) \delta: 6.20$ (d, $J=1.8 \mathrm{~Hz}, 1 \mathrm{H}, \mathrm{H}-6$ ), $6.42(\mathrm{~d}, J=1.8 \mathrm{~Hz}, 1 \mathrm{H}, \mathrm{H}-8), 7.81$ (d, $J=9.0 \mathrm{~Hz}, 2 \mathrm{H}$, H-2',6'), 6.95 (d, $\left.J=9.0 \mathrm{~Hz}, 2 \mathrm{H}, \mathrm{H}-3^{\prime}, 5^{\prime}\right), 5.41$ (d, $J=1.8$ $\mathrm{Hz}, 1 \mathrm{H}$, Rha I-H-1"), 4.23 (dd, $J=1.2,3.6 \mathrm{~Hz}, 1 \mathrm{H}$, Rha I-H-2"), 3.37 (dd, $J=3.0,9.0 \mathrm{~Hz}, 1 \mathrm{H}$, Rha I-H-3"), 3.33 (m, 1H, Rha I-H-4"), 3.39 (m, 1H, Rha I-H-5"), 0.96 (d, $J=6.0 \mathrm{~Hz} 1 \mathrm{H}$, Rha I-H-6"), 5.17 (d, $J=1.8 \mathrm{~Hz}, 1 \mathrm{H}$, Rha II-H-1"'), 4.12 (dd, $J=1.2,3.6 \mathrm{~Hz}, 1 \mathrm{H}$, Rha II-H-2"'), 3.79 (dd, $J=3.0,9.0 \mathrm{~Hz}, 1 \mathrm{H}$, Rha II-H-3"'), 3.82 (dd, $J=3.6$, $3.0 \mathrm{~Hz}, 1 \mathrm{H}$, Rha II-H-4"'), 3.58 (m, 1H, Rha II-H-5"'), 1.21 (d, $J=6.0 \mathrm{~Hz}, 1 \mathrm{H}$, Rha II-H-6"'), 4.54 (d, $J=7.8 \mathrm{~Hz}, 1 \mathrm{H}$, Glc I-H-1"''), 3.38 (m, 1H, Glc I-H-2"''), 3.45 (m, 1H, Glc I-H-3"'"), 3.32 (m, 1H, Glc I-H-4"''), 3.72 (m, 1H, Glc I-H-5"''), 4.51 (dd, $J=8.4,12.0 \mathrm{~Hz}, 1 \mathrm{H}$, Glc I-H-6"''), 4.61 (dd, $J=2.4,12.0 \mathrm{~Hz}, 1 \mathrm{H}$, Glc I-H-6"'"), 7.69 (br.s, 1H, H-2"'"'), 6.51 (d, $\left.J=8.4 \mathrm{~Hz}, 1 \mathrm{H}, \mathrm{H}-55^{\prime \prime \prime \prime)}\right), 7.68$ (dd, $J=8.4$, $1.8 \mathrm{~Hz}, 1 \mathrm{H}, \mathrm{H}-6^{\prime \prime \prime " ')}, 3.08$ (m, 1H, H-1"'"'), 5.19 (m, 1H, H-2"'"'), 1.71 (s, 1H, H-4"'"'), 1.63 (s, 1H, H-5"'"'); ${ }^{13} \mathrm{C}$ NMR (150 MHz, CD ${ }_{3} \mathrm{OD}$ ) $\delta: 159.8$ (s, C-2), 136.6 (s, C-3), 179.9 (s, C-4), 162.9 (s, C-5), 100.5 (d, C-6), 163.0 (s,
C-7), 95.4 (d, C-8), 158.0 (s, C-9), 107.7 (s, C-10), 122.5 (s, C-1'), 132.0 (d, C-2', 6'), 116.6 (d, C-3', 5'), 161.7 (s, C-4'), 103.6 (d, Rha I-C-1"), 70.8 (d, Rha I-C-2"), 72.2 (d, Rha I-C-3"), 73.2 (d, Rha I-C-4"), 72.1 (d, Rha I-C-5"), 17.7 (q, Rha I-C-6"), 99.0 (d, Rha II-C-1"'), 83.3 (d, Rha II-C-2"'), 72.1 (d, Rha II-C-3"'), 72.0 (d, Rha II-C-4"'), 70.9 (d, Rha II-C-5"'),18.1 (q, Rha II-C-6"'), 106.1 (d, Glc I-C-1"''), 75.2 (d, Glc I-C-2"''), 77.7 (d, Glc I-C-3"''), 72.5 (d, Glc I-C-4"'"), 75.7 (d, Glc I-C-5"'"'), 64.9 (t, Glc I-C-6"'"), 121.6 (d, C-1"'"'), 132.3 (d, C-2"'"'), 129.0 (d, C-3"'"'), 161.4 (d, C-4"'"'), 115.2 (d, C-5"'"'), 130.2 (d, C-6"'"'), 168.3 (s, C-7"'"'), 29.1 (t, C-1"'"'), 123.2 (d, C-2"'"'), 133.6 (d, C-3"'"'), 25.9 (q, C-4"'"'), 17.9 (q, C-5"'"'); IR (KBr) $v_{\text {max }}$ : 408, 2954, 2924, 2854, 1704, 1655, 1602, 1512, 1494, 1452, 1373, 1278, 1206, 1174, 1112, 1089, 1061, 1025, $973 \mathrm{~cm}^{-1}$; HR-ESI-MS calcd for $\mathrm{C}_{45} \mathrm{H}_{53} \mathrm{O}_{21}[\mathrm{M}+\mathrm{H}]^{+}$ 929.3079, found 929.3078 .

山奈酚-3,7-O- $\alpha-L$-二鼠李糖(2): 黄色无定型粉末. m.p. $201 \sim 205{ }^{\circ} \mathrm{C} ;{ }^{1} \mathrm{H}$ NMR (600 MHz, $\left.\mathrm{CD}_{3} \mathrm{OD}\right) \delta: 6.45$ (d, $J=3.0 \mathrm{~Hz}, 1 \mathrm{H}, \mathrm{H}-6), 6.72$ (d, $J=3.0 \mathrm{~Hz}, 1 \mathrm{H}, \mathrm{H}-8)$, 7.78 (d, $\left.J=13.2 \mathrm{~Hz}, 2 \mathrm{H}, \mathrm{H}-2^{\prime}, 6^{\prime}\right), 6.93$ (d, $J=13.2 \mathrm{~Hz}, 2 \mathrm{H}$, H-3',5'), 5.56 (br. s, 1H, Rha I-H-1"), 0.93 (q, $J=7.6 \mathrm{~Hz}$, $3 \mathrm{H}$, Rha I-H-6"), 5.39 (d, $J=1.8 \mathrm{~Hz}, 1 \mathrm{H}$, Rha II-H-1"'), 1.26 (q, $J=8.0 \mathrm{~Hz}, 3 \mathrm{H}$, Rha II-H-6"'); ${ }^{13} \mathrm{C}$ NMR (150 $\mathrm{MHz}, \mathrm{CD}_{3} \mathrm{OD}$ ) $\delta: 159.8$ (s, C-2), 136.5 (s, C-3), 179.8 (s, C-4), 163.0 (s, C-5), 100.5 (d, C-6), 163.5 (s, C-7), 95.6 (d, C-8), 158.1 (s, C-9), 107.5 (s, C-10), 122.4 (s, C-1'), 132.0 (d, C-2', 6'), 116.6 (d, C-3', 5'), 161.8 (s, C-4'), 103.5 (d, Rha I-C-1"), 72.1 (d, Rha I-C-2"), 71.9 (d, Rha I-C-3"), 73.6 (d, Rha I-C-4"), 73.1 (d, Rha I-C-5"), 17.7 (q, Rha I-C-6"), 99.8 (d, Rha II-C-1"'), 72.0 (d, Rha II-C-2"'), 71.7 (d, Rha II-C-3"'), 72.1 (d, Rha II-C-4"'), 71.3 (d, Rha II-C-5"'), 18.0 (q, Rha II-C-6"'); HR-ESI-MS calcd for $\mathrm{C}_{27} \mathrm{H}_{31} \mathrm{O}_{14}[\mathrm{M}+\mathrm{H}]^{+}$579.1714, found 579.1716. 以上数 据与文献[9]报道一致.

\section{References}

[1] Institute of Botany, Chinese Academy of Sciences and Institute of Materia Medica, Chinese Academy of Medical Sciences Flora Reipublicae Popularis Sinicae 1999, 18, 53 (in Chinese). (中国科学院植物所, 中国医学科学院药物所, 中国植物志, 1999, 18, 53.)

[2] Zhen, Y.-Z.; Zhang, Z.-K.; Ma, H.-W.; Bo, Y.-H. China Med. Her. 2016, 13, 44 (in Chinese).

(郑叶子, 张智宽，马宏文，卜亚恒，中国医药导报，2016，13， 44.)

[3] The State Administration of Traditional Chinese Medicine of the Chinese Herbal Medicine Editorial Board Chin. Mater. Med. 1999, 
24, 734 (in Chinese).

(国家中医药管理局编委会，中华本草，1999, 24, 734.)

[4] Fang, Z.-X.; Liao, C.-L. Flora of Enshi in Hubei Medicinal (Part ii), Hubei Science and Technology Press, Wuhan, 2006, 12, p. 721 (in Chinese).

(方志先, 廖朝林, 湖北恩施药用植物志 (下册), 湖北科学技术出 版社，武汉, 2006, 12, p. 721.)

[5] Li, H.-F.; Guan, X.-Y.; Yang, W.-Z.; Liu, K.-D.; Ye, M.; Sun, C.; Lu, S.; Gao, D.-A. Fitoterapia 2012, 83, 44.

[6] Zhen, Y.; Li, X.-W.; Gui, M.-Y.; Jin, Y.-R. China Pharm J. 2006, 43(3), 176 (in Chinese)
(郑芗，李绪文，桂明玉，金永日，中国药学杂志，2006，43(3)， 176.)

[7] Huang, S.; Zhou, X. L.; Wang, C.-J.; Wang, Y.-S.; Xiao, F.; Shan, L. H.; Guo, Z.-Y.; Weng, J. Phytochemistry 2013, 93, 154.

[8] Pang, Y.-W.; Wang, J.-D.; Xiang, S.-W.; Liu, C.-F.; Wang, X.-J. Nat. Prod. Res. Dev. 2012, 24, 1331 (in Chinese). (庞艳伟, 王继栋, 向文胜, 刘乾峰, 王相晶, 天然产物研究与开 发. 2012, 24, 1331.)

[9] Marzouk, M. M.; Kawashty, S. A.; Saleh, N. A. M.; Al-Nowaihi, A. S. M. Chem. Nat. Compd. 2009, 45,483 .

(Li, L.; Fan, Y.) 